\title{
El desempeño docente y la práctica inclusiva en el nivel
} superior ${ }^{1}$

Teaching performance and inclusive practice in higher education

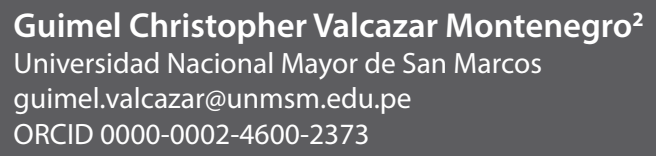

Citar como: Valcazar, G. (2020). El desempeño docente y la práctica inclusiva en el nivel superior. Desde el Sur, 12(2), pp. 437-452.

\section{RESUMEN}

La presente investigación tuvo como objetivo determinar la relación que existe entre el desempeño docente y la práctica inclusiva desde la percepción de los estudiantes de Mecánica Automotriz del Cetpro Benjamín Galecio Matos de Lima. Es un estudio de naturaleza aplicada, no experimental, de enfoque cuantitativo. La muestra seleccionada fue de 230 estudiantes de la institución, elegidos probabilísticamente. Se elaboraron dos encuestas con la intención de compilar información referida a las variables de estudio, una de 18 ítems para el desempeño docente y otra de 21 ítems para la práctica inclusiva.

Se realizó el contraste de la hipótesis por medio del rho Spearman $=, 793$ ( $p$-valor $=, 000<0,01)$ y se recabó suficiente información estadística para probar la hipótesis de investigación. El estudio concluye probando la existencia de la relación considerable y significativa entre el desempeño docente y la práctica inclusiva desde la percepción de los estudiantes de Mecánica Automotriz del Cetpro Benjamín Galecio Matos de Lima. Es decir, si hay un buen desempeño docente, este se verá reflejado con el aumento de una buena práctica inclusiva.

\footnotetext{
1 Investigación de tesis de maestría Desempeño docente y práctica inclusiva desde la percepción de los estudiantes de Mecánica Automotriz del Cetpro Benjamín Galecio Matos, Lima.

2 Licenciado en Administración y candidato a magíster en Educación con mención en Docencia Universitaria por la Universidad Nacional Mayor de San Marcos.
} 


\section{PALABRAS CLAVE}

Desempeño, inclusión, planificación, evaluación, aprendizaje

\section{ABSTRACT}

The objective of this research was to determine the relationship between teaching performance and inclusive practice from the perspective of students of automotive mechanics at CETPRO Benjamín Galecio Matos, in Lima. The study was of an applied nature, with a non-experimental design, employing a quantitative approach. The sample selected consisted of 230 students from the aforementioned educational institution, who were selected probabilistically. Two surveys were conducted, with the aim of compiling information regarding the study variables: one for teaching performance consisting of 18 items; and one consisting of 21 items for inclusive practice.

The hypothesis was tested using the Rho Spearman = .793 , ( $p$-value $=.000<0.01)$, with sufficient statistical information collected to test the research hypothesis. The study demonstrated the existence of a considerable and significant relationship between teaching performance and inclusive practice from the perspective of students of automotive mechanics at CETPRO Benjamín Galecio Matos, in Lima; in other words, where there is adequate teaching performance, this will be reflected in an increase in inclusive practice.

\section{KEYWORDS}

Performance, inclusive, planning, evaluation, learning

\section{Introducción}

El estudio contribuye a realzar los valores dentro de la comunidad educativa. La inclusión abre camino para una nueva forma de encarar las relaciones humanas desde el aula.

Las particularidades de entornos que demandan las entidades escolares son, sin dudar a dudas, los retos habituales de la inclusión. No obstante, los estudios al respecto aún son muy pocos y desconocen con precisión su cuantificación. Los dogmas de directores y docentes en cuanto a la inclusión, así como su relación con las experiencias que se llevan a cabo en las aulas, son muy diversas (Gelber, Treviño, González, Escribano y Ortega, 2019). Una de las insuficiencias principales del sistema educativo 
es la falta de comunicación efectiva que debe existir entre los profesores de las distintas instituciones educativas. Por lo tanto, se hallan restricciones en tres aspectos: las normas, la organización y los mismos profesores, lo que causa divergencias según las percepciones de los grupos de profesionales (García, Amezcua y Fuentes, 2019).

En España se llevó a cabo la implementación de políticas inclusivas con la finalidad de atender a los estudiantes con discapacidad. Al inicio se encontraron ciertas barreras, debido a la falta de conocimiento de las nuevas disposiciones institucionales $y$, además, por no querer aceptar los nuevos cambios y retos para el futuro (Pérez, Muñoz y Aravena, 2019).

Resalta la importancia de la inclusión como premisa en todas las acciones, dispositivos y disposiciones, considerando a los estudiantes que lo requieren. En los procesos de enseñanza-aprendizajes los docentes proceden de muchas formas inclusivas; de igual forma, efectúan acciones de tutorías estudiantiles mediante talleres o actividades grupales colaborativas. Es decir, los docentes del nivel superior desempeñan métodos científicos con el propósito de suscitar la interrelación inclusiva en el desarrollo de los procesos de aprendizaje, cognitivos y afectivos. También organizan acciones que permiten desarraigar todo acto de exclusión o discrepancia con el modelo pedagógico (Villavón, 2016).

Aún se mantiene en vigencia una apreciable cantidad de docentes que carecen de las prácticas o acciones inclusivas hacia la diversidad en todo aspecto. Estudios realizados a los docentes reflejan la escasa actitud positiva hacia la diversidad. Los datos señalan a la mala orientación y formación iniciales recibidas: «la educación intercultural surge como la necesidad de lograr desde la escuela actitudes transformadoras en un ambiente de convivencia en la colaboración, la tolerancia y el respeto ante la diversidad» (Pérez, 2016, p. 178).

Las políticas de carácter público acerca de la justicia social, equidad y educación dan pase a un enfoque de asimilación improcedente, apoyado en experiencias de absorción minoritaria y desvalorización de las diferencias en especial (Ocampo y López, 2019). En este sentido, se apuesta a un enfoque relacional que reconoce la presencia de diversos grupos y etnias sociales, que constan de inmensa desigualdades (González, 2016).

Diversos estudios dan a conocer los niveles del desempeño docente y la organización de actividades de 9,0 . En cuanto a si resuelve conflictos en el aula de 9,6, aplica el código de convivencia de 8,6 e identifica los niveles del sistema educativo de 9,0. Sobre el contraste de hipótesis, indicó un valor $r S=, 502$ ( $p$-valor $<, 01$ ). Se concluyó que es moderada la relación entre el desempeño docente y el proceso de enseñanza-aprendizaje. Por su 
parte, Mautino (2018) obtuvo datos descriptivos en la variable inclusión al referir el nivel bueno de 6,2\%, regular de 55,4\% y deficiente de 38,5\%. En desempeño docente: nivel bueno $9,2 \%$, regular $64,6 \%$ y deficiente $26,2 \%$. Además, rS = ,616 ( $p$-valor <,05).

En este sentido, se observa una estrecha relación entre la actitud docente y las prácticas educativas inclusivas. Goldsmith (2016) alcanzó valores correlacionales de $\mathrm{rS}=, 364$, entre el uso de prácticas educativas inclusivas y la actitud del profesorado hacia la inclusión. Asimismo, otra investigación realizada con 253 estudiantes elegidos al azar registró datos del desempeño docente de 57,7\% para el nivel previsto. El 23,7\% indicó estar en proceso y el 8,3\%, en inicio. Su contraste fue de $r S=, 812$ ( $p$-valor $<0,01)$. Se concluyó que existe correlación alta y positiva entre las variables de desempeño docente y el logro de aprendizaje (Rodríguez, 2017).

En las diversas investigaciones ejecutadas se puede apreciar la gradualidad de las relaciones e interrelaciones que tiene el desempeño docente con diversas variables, como la motivación, el rendimiento académico, las prácticas inclusivas, el bullying y los aprendizajes (Espinoza, Vilca y Pariona, 2014; Aguirre, 2015; Barreto, 2015).

\section{Desempeño docente}

El desempeño docente incluye las funciones, tareas y actividades que se realizan desde la propia concepción del docente, basada en ideas o teorías, además de los saberes propios de su formación profesional

Los procesos de enseñanza-aprendizaje son complejos y, por ese motivo, se involucran en ellos aspectos motivacionales y dinámicas constantes para adaptarse a los cambios y requerimientos del sistema educativo (Lavados, 2015). Asimismo, el desempeño docente considera cinco áreas: la planificación de las actividades docentes, la ejecución de las actividades docentes, la evaluación de los aprendizajes, la evaluación de la práctica pedagógica y el compromiso con el proyecto educativo institucional. Las cinco áreas se detallan a continuación.

La planificación es prever las acciones para el futuro. Preparar el material de clase brinda mayor posibilidad de conseguir un mejor desempeño en el aula, considerando lo que se establece en el sílabo y currículo, además de lo que el estudiante requiere para su formación profesional. La ejecución de las actividades consiste en ejecutar lo antes programado, poner en marcha todos los mecanismos, las herramientas pedagógicas, los procesos y el uso de tecnologías, así como el contexto donde se llevarán a cabo. En la evaluación de los aprendizajes el docente tiene la capacidad de observar metódicamente a sus estudiantes. Recopila, 
examina y compara sus logros para medir el avance de sus requerimientos. A la vez, redirige sus estrategias y, si se requiere, realiza retroalimentación. La planificación educativa es inherente a la labor docente, así como impartir conocimientos y evaluar los aprendizajes con profesionalismo. Todas las sesiones de clases, sílabos y programas se organizan teniendo en consideración la programación curricular para cada ciclo y nivel. Impartir conocimiento no es nada fácil, pues en esta tarea está inmerso el compromiso con los estudiantes de lograr sus aprendizajes. Para ello se emplean diversas técnicas y estrategias de enseñanza, con el propósito de impartir conocimiento que cubra de alguna forma sus requerimientos propios (Stronge, 2015).

En el tema de la evaluación pedagógica, las prácticas pedagógicas son vitales en las distintas instancias y los procesos que participan en la enseñanza-aprendizaje. La idea es tener claro el progreso que se va logrando. $\mathrm{Y}$, por último, es necesario el compromiso con el proyecto educativo institucional, la lealtad con la entidad. Así, el docente colabora con los demás docentes y la comunidad educativa en la realización de los proyectos institucionales, además de valorar la misión y la visión, y generar vías de comunicación orientadoras (Lavados, 2015). La evaluación no es tan simple de realizar, pues entran en juego muchos factores de valores pedagógicos. Los nuevos enfoques psicopedagógicos consideran la evaluación cuantitativa y cualitativa de los aprendizajes (Hortigüela, Pérez-Pueyo y González-Calvo, 2019).

Por otro lado, la evaluación del aprendizaje recopila, examina y compara el logro que va obteniendo de sus alumnos. El entorno donde se realizan los aprendizajes debe crear el contexto que facilite los aprendizajes, mediante el uso de recursos y material didáctico para afianzar los conocimientos. Asimismo, se requiere proveer un ambiente seguro de trabajo en equipo, de respeto e inclusión centrada en los estudiantes, para asegurar el proceso de enseñanza-aprendizaje (Stronge, 2015).

El proceso de evaluación del desempeño docente también se genera en la educación superior, en un nivel educativo que debe medir la productividad intelectual académica. Por lo tanto, esta evaluación se centra en la investigación, no obstante que el desenvolvimiento del docente ocupa gran parte de su tiempo en preparar su clase y los materiales didácticos. La evaluación debe estar relacionada con la toma de decisiones y la ejecución de los cambios para destacar las debilidades reveladas (Lavados, 2015). La evaluación formativa plantea una noción constructivista donde el estudiante organiza sus aprendizajes por medio de acciones constructivas de logros. Esta forma de enjuiciar los aprendizajes permite al docente conocer y reconocer los errores que cometen los estudiantes, y para tal 
efecto toma decisiones para enmendarlos, como parte de su desempeño profesional (Pasek y Mejía, 2017).

La preparación y la capacitación profesionales salvaguardan con responsabilidad la ética docente, respetando las políticas institucionales (Stronge, 2015). Es importante perfeccionar los aprendizajes, mediante la capacitación constante y la adquisición de nuevas competencias pedagógicas que mejoren sus destrezas (Pérez, Enrique, Carbó y González, 2017).

La conducta y el accionar del docente deben crear valores en los estudiantes, con la posibilidad de que sean útiles para la sociedad. Los docentes en su labor cotidiana ejecutan actividades de exposición pública con otras instituciones e intercambio cultural. Estas acciones favorecen al desarrollo integral de las personas y fortalecen las capacidades de sentido crítico e inteligencia emocional (Alcalde, 2015). El reconocimiento del trabajo digno y de capacitación continua del docente lo predispone para la comunicación acertada, la convivencia, el desarrollo de la institución y la sustentabilidad de la organización. Todo este accionar se complementa con las instalaciones y la infraestructura que ayuden a fortalecer su trabajo: aulas acondicionadas y equipadas con multimedia (Cuadrado, 2014).

Estudios realizados acerca de la relación entre el compromiso organizacional y el desempeño, con una muestra de 83 docentes, elegidos probabilísticamente, dan a conocer $r s=, 724(p=0,000<0,05)$ (Estrada y Mamani, 2020). La existencia de diferencias reveladoras entre el desempeño docente y varias poblaciones de estudio, mediante un estudio descriptivo comparativo, afirman la existencia de diferencias reveladoras en el desempeño docente en ambos grupos comparados $(U=178,50, Z=-5,301$, $\mathrm{p}<$,001) (Álvarez, 2020).

De la misma forma, Espinoza (2019) efectuó el estudio acerca de las fortalezas y debilidades del desempeño docente, de enfoque mixto, fenomenológico, hermenéutico y de método analítico-sintético. Se aplicaron cuestionarios de encuestas a 216 estudiantes de educación inicial. La información recabada indicó las fortalezas de flexibilidad, comprensión y puntualidad, mientras que las debilidades fueron el uso de adecuadas didácticas, los recursos informáticos y las plataformas virtuales.

Un estudio realizado en diversas universidades privadas de BarranquiIla, con la participación de 107 docentes de nivel universitario, elegidos aleatoriamente, aplicó una encuesta de 30 ítems. Se llegó a la conclusión de que los docentes son líderes en la formación académica y están dotados de habilidades que permiten proveer a sus estudiantes desarrollar capacidades cooperativas, motivadoras, de toma de decisiones y de relaciones interpersonales (Niebles-Núñez, Hoyos-Babilonia y De-La-Ossa-Guerra, 2019). 


\section{Práctica inclusiva}

El enfoque de la educación inclusiva llevada a la práctica docente inclusiva se basa en la valoración de la diversidad multicultural, multilingüista y otros enfoques, con la finalidad de vigorizar los valores y la autoestima de los estudiantes, y mejorar sus perspectivas de vida, siempre centrado en la labor docente y en brindar apoyo a todos los estudiantes (Minedu, 2019).

Mediante una perspectiva crítica e inclusiva de las prácticas lectoras contemporáneas, inspecciona la lectura desde una práctica político-cultural, como parte de la justicia ciudadana emancipadora que critica reflexivamente las desilusiones intelectuales y las políticas imprecisas de la educación. La práctica de la lectura es una herramienta valiosa que garantiza la formación inclusiva, el derecho de cada persona y la justicia social (Ocampo y López, 2020). Existe una fisura entre la teoría y la práctica para volcar el discernimiento inclusivo en los estudiantes, en cuanto a la separación de dos grandes brechas de la práctica inclusiva. Por un lado, son las estrategias usadas por los maestros $y$, por el otro, es el reconocimiento acerca de lo que significa en sí mismo la inclusión (Moliner, Arnaiz y Sanahuja, 2020).

Es primordial la promulgación de resoluciones o dictámenes acerca de la inclusión en las instituciones de nivel superior, que permiten el acceso a los diferentes horizontes de lo que las aulas exigen actualmente. A nivel internacional hay abundante material escrito acerca de la inclusión, pero en Latinoamérica aún falta mucho por avanzar (Clavijo y Bautista, 2020). La aplicación de técnicas de flujogramas y la revisión organizada de documentación de contenido especializado permiten la correspondencia entre los diversos factores que surgen de la práctica pedagógica en el aula, como la competencia docente y la práctica inclusiva, que fortifican el proceso de inclusión en los estudiantes (Royo, Petit, Salazar y Rada, 2019).

Las prácticas inclusivas e interculturales destinadas al alumnado, desde una perspectiva etnográfica, entrevistas y análisis documentario, mostraron la existencia de diferencias muy resaltantes en el uso de métodos pedagógicos y solo continúan con la práctica tradicional. Las prácticas inclusivas interculturales son insuficientes (Rosado, 2019).

Un análisis del aprendizaje cooperativo para la educación inclusiva, con el objetivo de optimar la atención a la variedad inclusiva en los estudiantes con necesidades concretas de programas, métodos, estrategias de enseñanza, técnicas didácticas, formas de evaluación e información documentaria de 2011 a 2017, mostró la atención a la diversidad e inclusión en los espacios de aprendizaje, y la formación docente en la preparación de material educativo atendiendo a los estudiantes que requieren 
de observación inclusiva (Perlado, Muñoz y Torrego, 2019). La enseñanza inclusiva en España refiere que la atención a la diversidad no se toma en consideración a un conjunto de estudiantes específicamente, sino a la manera en que se debe educar a todos (Toboso, Ferreira, Díaz, Fernández, Villa y Gómez, 2012).

«La inclusión o la educación inclusiva no es otro nombre para referirse a la integración de alumnos con necesidades educativas especiales. Implica un enfoque diferente para identificar e intentar resolver las dificultades que aparecen en los centros» (Echeita, 2014, p. 94). Es la atención a la diversidad en todo aspecto, considerando a todos los estudiantes en general, indistintamente de las necesidades educativas especiales. Echeita la considera «una cuestión de valores, una opción sobre la educación que queremos para nuestros hijos y sobre el tipo de sociedad en la que nos gustaría vivir» (2014, p. 95). El investigador contempla tres dimensiones de la práctica inclusiva: cultura inclusiva, prácticas inclusivas y políticas inclusivas. Refiere que "gracias a la cultura de los centros educativos se producen cambios en las políticas y en las prácticas, que pueden mantenerse y transmitirse a los nuevos miembros de la comunidad escolar» (Echeita, 2014, p. 156).

La cultura inclusiva tiene su pedestal en la enseñanza de los valores inclusivos y los hace extensivos en toda la comunidad educativa, específicamente entre los estudiantes y profesores. Es el soporte que debe predominar, el apoyo que se le debe brindar a todo estudiante de parte del maestro y la institución educativa. Echeita indica que «está orientada hacia la creación de una comunidad escolar segura, acogedora, colaboradora y estimulante, en la que cada uno es valorado como el fundamento primordial para que todo el alumnado tenga mayores niveles de logro» (2014, p. 157). Las prácticas inclusivas requieren la unificación de todos los docentes para diseñar experiencias de aprendizaje que prevalezcan los obstáculos de exclusión. Asimismo, se necesita diseñar tácticas que ayuden a la integración de todos los estudiantes en las prácticas inclusivas y sus aprendizajes dentro de la institución educativa. A su vez, las políticas inclusivas apoyan todas las acciones o actividades que realizan las instituciones educativas para atender los requerimientos de los estudiantes inclusivos. Echeita refiere que «esta dimensión pretende asegurar que la inclusión esté en el corazón del proceso de innovación, empapando todas las políticas, para que mejore el aprendizaje y la participación de todos los estudiantes» (2014, p. 157). 


\section{La inclusión en la educación superior}

El incremento de estudiantes del nivel superior llegó a 1247746 en 2017. Sin embargo, no ha logrado hacer cambios significativos en la posición sociocultural de los estudiantes y en otros factores relegados de la sociedad. La Tercera Conferencia Regional de Educación Superior en América Latina y el Caribe, celebrada en junio de 2018, destaca la importancia de la comisión de Educación Superior, diversidad cultural e interculturalidad en América Latina. La conferencia dio iniciativas con el fin de enfocar las prácticas inclusivas, en el nivel superior, y promovió proyectos orientados a la inclusión de estudiantes en condición de discapacidad y demás grupos humanos rezagados. A pesar de los cambios en sus protocolos de matrícula, adaptación curricular, titulación y edificación, no se ha podido minimizar la brecha del enfoque intercultural, de acuerdo con el perfil de los estudiantes que provienen de diferentes áreas geográficas del país (Mineduc, 2017).

\section{La interculturalidad}

Es «un enfoque que busca resguardar y valorar las diferentes culturas; entre ellas destacan los pueblos indígenas y la población migrante» (Mineduc, 2017, p. 13). En la acción intercultural se dinamiza la interrelación entre las diferentes culturas, mediante un diseño de gestión transversal que busca concentrar los procesos educativos con la perspectiva intercultural y bilingüe de los pueblos indígenas.

\section{La discapacidad}

El Servicio Nacional de Discapacidad (Sinadis) define a las personas discapacitadas como aquellas «que, en sus condiciones de salud física, psíquica, intelectual, sensorial u otras, al interactuar con diversas barreras contextuales, actitudinales y ambientales, presentan restricciones en su participación plena y activa en la sociedad» (Mineduc, 2017).

\section{Materiales y métodos}

El estudio fue de tipo aplicado. El investigador tuvo la intención del empleo práctico y aplicativo en el desempeño docente y los procesos de inclusión de los estudiantes en la educación, según la percepción de los estudiantes de Mecánica Automotriz del Cetpro Benjamín Galecio Matos de Lima.

El nivel empleado fue correlacional, debido a que su propósito fue determinar el nivel de relación entre el desempeño docente y la práctica inclusiva en estudiantes del nivel superior técnico productivo en la mencionada institución. 
Fue de diseño no experimental transversal, por lo que no se realizó la manipulación de las variables de estudio. Además, se ejecutó solo una medición para obtener información de las variables observadas.

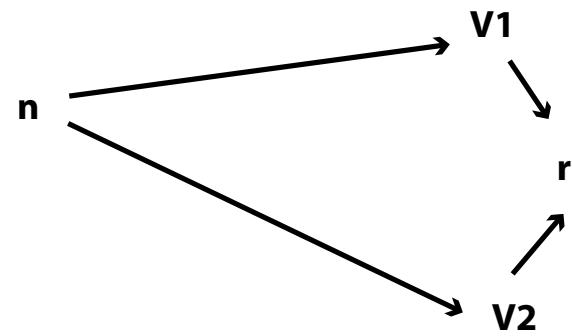

Donde:

$\mathrm{n}$ : Muestra

V1: Desempeño docente

$r$ : Coeficiente de correlación

V2 : Práctica inclusiva

\section{Población}

La población fue de 536 y su muestra fue de 230 estudiantes de Mecánica Automotriz del Cetpro Benjamín Galecio Matos de Lima.

\section{Proceso de prueba de hipótesis}

Se realizó el estudio de la población, teniendo en consideración los diferentes cursos (Suspensión de Frenos, Sistema de Transmisión, Motores a Gasolina, Motores Diésel, Sistema Automotriz y Sistema Electrónico), para luego establecer la similitud de esta población. De este modo, se determinó la muestra representativa para el estudio. Se diseñaron dos instrumentos, uno para cada variable, en principio con 18 ítems para el desempeño docente y 21 ítems para la práctica inclusiva. La fiabilidad de los instrumentos fue de, 939 y, 935 , respectivamente, y se empleó el coeficiente alfa de Cronbach. Los instrumentos fueron validados por juicio de un experto.

Se realizó la prueba de normalidad Kolmogórov-Smirnov y se determinó que los datos no se ajustaban a una distribución normal. Debido a ello, se optó por usar el estadístico rho de Spearman, para el contraste de las hipótesis.

\section{Resultados}

Con una muestra de 230 estudiantes de Mecánica Automotriz del Cetpro Benjamín Galecio Matos de Lima, se halló que el 36,5\% expresó un nivel medio, mientras que el $63,5 \%$ señaló un nivel alto. Para la práctica inclusiva el $18,3 \%$ manifestó un nivel medio y el $81,7 \%$ expresó un nivel 
alto. El valor obtenido mediante la prueba de rho de Spearman $=, 793$ ( $p$-valor <,01). En tal sentido, se concluye que, al haber suficiente evidencia estadística, se rechazó la hipótesis nula. Por lo tanto, se probó la existencia de correlación entre el desempeño docente y la práctica inclusiva, con margen de error menor al 1\% muy significativo. También se probaron todas las hipótesis específicas planteadas en el estudio.

\section{Discusión}

Se probaron todas las hipótesis y se reveló la relación media y considerable entre las variables de estudio, significativas y positivas, así como su valor para el desempeño docente y la práctica inclusiva $r S=, 793$ ( $p$-valor $<0,1)$. Se evidenció la importancia de la planificación de las sesiones de clases y los contenidos, conjuntamente con las estrategias de enseñanzaaprendizaje, el material didáctico y las evaluaciones constantes para ver el logro de los estudiantes y reestructurar si fuera necesario. Por último, el docente se identifica con el desarrollo profesional de sus alumnos.

De la misma forma, se evidenció la relación positiva, media y significativa de $r S=, 720$ ( $p$-valor $<0,1)$ entre la planificación de las actividades docentes y la práctica inclusiva. Se acreditó la relevancia de dosificar los contenidos y el tiempo de las sesiones de clase, la entrega oportuna del material y la información a los estudiantes de sus logros y avances de aprendizajes.

De la misma forma, se evidenció la relación positiva, media y significativa de $\mathrm{rS}=, 721$, ( $\mathrm{p}$-valor $<0,1$ ) entre la ejecución de las actividades docentes y la práctica inclusiva. Se confirmó la relevancia del uso de la enseñanza activa con nuevas estrategias, el uso de material tecnológico y el reforzamiento continuo.

En efecto, se evidenció la relación positiva, media y significativa de $r S=, 708$ ( $p$-valor $<0,1)$ entre la evaluación de los aprendizajes y la práctica inclusiva. Asimismo, se acreditó la notabilidad de la evaluación permanente y de diversas formas para la retroalimentación, si fuera el caso.

Se evidenció la relación positiva, considerable y significativa de $r S=, 783$ ( $p$-valor $<0,1)$ entre la evaluación de la práctica pedagógica y la práctica inclusiva. Se acreditó la solidez de la comunicación entre el docente y los estudiantes para la realización de las prácticas estructuradas, y la capacitación permanente del profesional docente.

De la misma forma, se evidenció la relación positiva, media y significativa de $r S=, 810$ ( $p$-valor $<0,1)$ entre el compromiso con el proyecto educativo institucional y la práctica inclusiva. Se acreditó la importancia de la identificación docente con la institución mediante su misión y visión, 
manteniendo la ética profesional, y el involucramiento y desarrollo personal de sus estudiantes.

Vistos los resultados obtenidos, estos hallan semejanza con Espinosa (2014), cuyo objetivo fue determinar la relación entre el desempeño docente y el proceso de enseñanza-aprendizaje, con una muestra de 53 estudiantes. Los resultados para el desempeño docente (escala del 1 al 10) son los siguientes: el docente organiza las actividades, 9,0; resuelve conflictos en el aula, 9,6; aplica el código de convivencia, 8,6; identifica los niveles del sistema educativo, 9,0. La prueba de hipótesis tiene un valor de $r S=, 502$ ( $p$-valor $<, 01)$, y se concluye una relación moderada entre ambas variables.

Del mismo modo, Barreto (2015) tuvo el objetivo fue determinar la relación entre el desempeño docente y el desarrollo de las capacidades en gestión de aprendizaje, con una muestra de 80 estudiantes del VI ciclo. El resultado para el desempeño docente, respecto al grado de planificación, indica lo siguiente en el proceso E-A: el 55\% siempre, el 30\% casi siempre y el $15 \%$ a veces. En la evaluación de los aprendizajes, el $45 \%$ siempre, el $45 \%$ casi siempre y el $10 \%$ a veces. En el grado de dominio de los contenidos, el $50 \%$ siempre, el $35 \%$ casi siempre y el $15 \%$ a veces. Su valor de contraste fue $\mathrm{rS}=, 778$ ( $\mathrm{p}$-valor $<0,05)$. De esta manera se probó la hipótesis que referiría la influencia entre el desempeño docente y las capacidades en gestión empresarial y marketing.

Asimismo, se encuentra analogía con Piña (2010), que tuvo el objetivo de determinar la relación existente entre el desempeño docente con las habilidades del estudiante y el rendimiento académico. Los datos obtenidos para el desempeño docente fueron: el $47,3 \%$ de los maestros tenían un nivel muy bueno; el $43,8 \%$, bueno, y el $8,9 \%$, deficiente. En cuanto al rendimiento académico, los estudiantes indicaron $52,0 \%$ en un nivel bueno, el $38,8 \%$ nivel bueno y el $9,2 \%$ deficiente. Para el contraste de hipótesis se tuvo un valor de $t=, 630$ ( $p$-valor $<0,05$ ). Se comprobó la hipótesis alterna indicando la influencia del desempeño docente y el rendimiento académico.

El estudio mostró analogía asimismo con Mautino (2018), cuyos resultados descriptivos de la variable inclusión indicaron un nivel bueno de $6,2 \%$, un nivel regular de $55,4 \%$ y un nivel deficiente de $38,5 \%$. Asimismo, para el desempeño docente detallaron el nivel bueno en 9,2\%, regular en $64,6 \%$ y deficiente en $26,2 \%$. Su contraste fue $r S=, 616$, significativo con $p$-valor $<, 05$. En tal sentido, se probó la relación positiva moderada entre la inclusión educativa y el desempeño docente. 
Además, tuvo similitud con el estudio realizado por Goldsmith (2016), cuyo propósito fue determinar la relación entre la actitud docente y el uso de prácticas educativas inclusivas, con una muestra de 183 docentes y la aplicación de una encuesta. Se usó un valor de $r S=, 364$ para la práctica educativa inclusiva y la actitud del profesorado hacia la inclusión.

El estudio también halla correspondencia con la investigación de Rodríguez (2017), que se planteó el objetivo de determinar la relación entre el desempeño docente de comunicación con el logro de aprendizaje. Su muestra fue de 253 estudiantes y sus datos se compilaron por una encuesta. El resultado para el desempeño docente fue: el $57,7 \%$ tuvo un nivel previsto, el $23,7 \%$ referenció estar en proceso y el $8,3 \%$ indicó estar en inicio. Su contraste de hipótesis arrojó el $r S=, 812$ ( $p$-valor $<0,01$ ).

\section{Contribución del autor}

Guimel Christopher Valcazar Montenegro ha participado en la elaboración, la compilación de datos, la redacción y el consentimiento de la versión final del artículo.

\section{Fuente de financiamiento}

Autofinanciado.

\section{Conflicto de interés}

El autor declara no tener compromisos de interés.

\section{REFERENCIAS BIBLIOGRÁFICAS}

Aguirre Espíritu, M. N. (2015). Desempeño docente y rendimiento académico en el área de comunicación en estudiantes del $V$ ciclo de primaria de la institución educativa particular Nuestra Señora de las Mercedes. Rímac, 2014 (tesis de maestría). Universidad César Vallejo, Lima.

Alcalde, I. (2015). Docentes del siglo XXI: retos y habilidades clave. Recuperado de https://www.linkedin.com/pulse/docentes-del-siglo-xxi-retosy-habilidades-clave-ignasialcalde

Barreto Bardales, I. (2015). Desempeño docente y desarrollo de las capacidades en gestión empresarial y marketing en estudiantes de la Escuela Superior Tecnológica, Sencico (tesis doctoral). Universidad San Martín de Porres, Lima. 
Campoverde Gómez, M. (2014). Desempeño docente en el proceso de enseñanza aprendizaje en el nivel de Educación Básica de la Unidad Educativa Belisario Quevedo de la ciudad de Pujilí, provincia de Cotopaxi, año lectivo 2013-2014 (tesis de maestría). Universidad Técnica Particular de Loja, Cotopaxi.

Clavijo Castillo, R. y Bautista Cerro, M. (2020). La educación inclusiva. Análisis y reflexiones en la educación superior ecuatoriana. Alteridad, 15(1), pp. 113-124. DOI: org/10.17163/alt.v15n1.2020.09

Cuadrado, R. (2014). 4to. Simposio Internacional de Investigación de Ciencias Económicas, Administrativas y Contables. Recuperado de http:// www.unilibre.edu.co/bogota/ul/noticias/noticias-universitarias/26694-to-simposio-internacional-de-investigacion-en-ciencias-economicasadministrativas-y-contables

Echeita Sarrionandia, G. (2014). Educación para la inclusión o educación sin exclusiones. Bogotá: Ediciones de la U.

Espinoza Almendras, J., Vilca Alcántara, C. y Pariona Criales, J. (2014). El desempeño docente y el rendimiento académico en el curso de aritmética: conjuntos, lógica proposicional del cuarto grado de secundaria de la Institución Educativa Pamer de Zárate, San Juan de Lurigancho, Lima, 2014 (tesis de maestría). Universidad Nacional de Educación Enrique Guzmán y Valle, Lurigancho-Chosica.

García Domingo, M., Amezcua Aguilar, T. y Fuentes Gutiérrez, V. (2019). El reto de la educación inclusiva: Elementos implicados y propuestas de mejora. Prisma Social, 4(27), pp. 40-64.

Gelber, D., Treviño, E., González, A., Escribano, R. y Ortega, L. (2019). Del dicho al hecho: creencias y prácticas inclusivas en establecimientos y aulas escolares en Santiago. Perspectiva Educacional. Formación de Profesores, 58(3), pp. 73-101. DOI: 10.4151/07189729-Vol.58-Iss.3-Art.967

Goldsmith Sánchez, H. (2016). Actitud docente y prácticas educativas inclusivas en estudiantes con necesidades educativas especiales en instituciones educativas públicas de la UGEL, Casma, 2014 (tesis de maestría). Universidad Peruana Unión, Lima.

Gonzáles Gil, F. (2016). Percepciones del profesorado sobre la inclusión: estudio preliminar. Revista Electrónica Interuniversitaria de Formación del Profesorado, 19(3), pp. 11-24.

Hortigüela, D., Pérez-Pueyo, A. y González-Calvo, G. (2019). Pero... ¿a qué nos referimos realmente con la evaluación formativa y compartida? Confusiones habituales y reflexiones prácticas. Revista lberoamericana de Evaluación Educativa, 12(1), pp. 13-27. DOI: http://dx.doi.org/10.15366/ riee2019.12.1.001 
Irigoyen, J., Acuña, K. y Jiménez, M. (2011). Evaluación de desempeños académicos. Sonora: Universidad de Sonora.

Lavados Montes, I. (2015). Evaluación del desempeño docente y calidad de la docencia universitaria. Santiago de Chile: Ministerio de Educación de Chile, Mineduc.

Martínez Figueira, E., Páramo Iglesias, B. y Claudino Necho, E. (2015). Desafíos actuales a la inclusión: un estudio de caso en un aula de preescolar portuguesa. Actualidades Investigativas en Educación, 15(1), pp. 1-18. DOI: dx.doi.org/10.15517/aie.v15i1.17736

Mautino Soria, V. (2018). Inclusión educativa y desempeño docente en las instituciones educativas públicas, Red 08, UGEL 02, Rímac, 2018 (tesis de maestría). Universidad César Vallejo, Lima.

Minedu (2012). Marco del buen desempeño docente. Lima: Ministerio de Educación del Perú.

Minedu (2019). Plan de tutoría, orientación educativa y convivencia escolar 2019. Lima: Ministerio de Educación del Perú. Recuperado de https:// www.ugel05.gob.pe/documentos/3_12marzo2019_PLAN_DE_TOECE_2019_IE_versi\%C3\%B3n_PDF.pdf

Mineduc (2017). Bases para la construcción de una política inclusiva en educación superior. Santiago de Chile: División de Educación Superior, Ministerio de Educación de Chile.

Moliner García, O., Arnaiz Sánchez, P. y Sanahuja Ribés, A. (2020). Rompiendo la brecha entre teoría y práctica: ¿qué estrategias utiliza el profesorado universitario para movilizar el conocimiento sobre educación inclusiva? Educación XXI, 23(1), pp. 173-195. DOI: 10.5944/educXX1.23753

Niebles-Núñez, W., Hoyos-Babilonia, L. y De-La-Ossa-Guerra, S. (2019). Clima Organizacional y Desempeño Docente en Universidades Privadas de Barranquilla. Saber, Ciencia y Libertad. 14, (2), pp. 283-294. DOI:https:// doi.org/10.18041/2382-3240/saber.2019v14n2.5893.

Ocampo González, A. y López Andrada, C. (2020). Transformando los espacios de lectura. Una perspectiva crítica e inclusiva de las prácticas lectoras contemporáneas. Educare Electronic Journal, 24(1), pp. 1-21. DOI: http:// doi.org/10.15359/ree.24-1.11

Pasek de Pinto, E. y Mejía, M. (2017). Proceso general para la evaluación formativa del aprendizaje. Revista Iberoamericana de Evaluación Educativa, 10(1), pp. 177-193. DOI: https://doi.org/10.15366/riee2017.10.1.009

Pérez Poquet, M., Muñoz Villalobos, P. y Aravena Rivas, V. (2019). Innovación educativa en contextos inclusivos de educación superior. En M. Arancibia Muñoz, R. Romero Alonso y C. Maregatti Solano (Coords), Programa de inclusión para actores de educación superior técnico-profesional (pp. 101114). Barcelona: Ediciones Octaedro S. L. 
Pérez, M., Enrique, J., Carbó, J. y González, M. (2017). La evaluación formativa en el proceso enseñanza aprendizaje. Edumecentro, 9(3), pp. 263-283. Recuperado de http://www.revedumecentro.sld.cu/index.php/edumc/ article/view/989/pdf_261

Perlado Lamo de Espinoza, I., Muñoz Martínez, Y. y Torrego Seijo, J. (2019). Implicaciones de la formación del profesorado en aprendizaje cooperativo para la educación inclusiva. Profesorado. Revista de Currículum y Formación de Profesorado, 23(4), pp. 128-151. DOI: 10.30827/profesorado.v23i4.9468

Piña Sangama, R. (2010). El desempeño docente y su relación con las habilidades del estudiante y el rendimiento académico en la universidad particular de lquitos, año 2010 (tesis doctoral). Universidad Nacional Mayor de San Marcos, Lima.

Rodríguez Hurtado, M. (2017). El desempeño docente y el logro de aprendizaje en el área de comunicación en las instituciones educativas de Chaclacayo en el 2015 (tesis de maestría). Universidad Nacional Mayor de San Marcos, Lima.

Rosado Holguín, G. (2019). Prácticas inclusivas e interculturales destinadas al alumnado recién llegado de otros países a Cataluña. Scientific, 4(14), pp. 163-181. DOI: doi.org/10.29394/Scientific.issn.2542-2987.2019.4.14.8.163181

Royo Peña, H., Petit Torres, E., Salazar Caballero, Y. y Rada Tovar, A. (2019). Innovación teórica para analizar el proceso de inclusión estudiantil desde la práctica pedagógica. Zona Próxima, 31, pp. 56-86.

Stronge, J. (2015). Sistema de evaluación del desempeño de docentes. Quito: Asociación Americana de Escuelas en Sudamérica.

Toboso, M., Ferreira, M., Díaz, V., Fernández, E., Villa, N. y Gómez, C. (2012). Sobre la educación inclusiva en España: políticas y prácticas. Intersticios. Revista Sociológica de Pensamiento Crítico, 6(1), pp. 279-295. Recuperado de http://riberdis.cedd.net/handle/11181/3525

Villalón García, G. (2016). Enfoques actuales sobre la formación del maestro en Cuba. Santiago de Cuba: Cátedra. 